\title{
SINGULAR VARIATION OF DOMAINS AND CONTINUITY PROPERTY OF EIGENFUNCTION FOR SOME SEMI-LINEAR ELLIPTIC EQUATIONS
}

\author{
Shin Ozawa And Susumu RoppongI
}

\section{Introduction}

Let $M$ be a bounded domain in $\boldsymbol{R}^{3}$ with smooth boundary $\partial M$. Let $w$ be a fixed point in $M$. By $B(\varepsilon ; w)$ we denote the ball of center $w$ with radius $\varepsilon$.

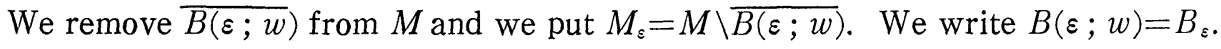

Fix $k \geqq 0$ and $p \in(1,5)$. We put

$$
\lambda(\varepsilon)=\inf _{X_{\varepsilon}}\left(\int_{M_{\varepsilon}}|\nabla u|^{2} d x+k \int_{\partial M_{\varepsilon}} u^{2} d \sigma\right),
$$

where

$$
X_{\varepsilon}=\left\{u \in H^{1}\left(M_{\varepsilon}\right):\|u\|_{L^{p+1}{ }_{\left(M_{\varepsilon}\right)}}=1, u=0 \text { on } \partial M, u \geqq 0 \text { in } M_{\varepsilon}\right\} .
$$

Then, we know that there exists at least one solution $u_{\varepsilon}$ which attains $(1.1)_{\varepsilon}$. It satisfies

$$
\begin{aligned}
& -\Delta u_{\varepsilon}=\lambda(\varepsilon) u_{\varepsilon}^{p} \quad \text { in } \quad M_{\varepsilon} \\
& \frac{\partial u_{\varepsilon}}{\partial \nu_{x}}+k u_{\varepsilon}=0 \quad \text { on } \quad \partial B_{\varepsilon} \\
& u_{\varepsilon}=0 \quad \text { on } \partial M .
\end{aligned}
$$

Here $\partial / \partial \nu_{x}$ denotes the derivative along the exterior normal direction.

One of the main results of this paper is the following.

THEOREM 1. Fix $p \in(1,5)$. Then, there exists a constant $C$ independent of $\varepsilon$ such that

$$
\sup _{u_{\varepsilon} \in S_{\varepsilon}} \sup _{x \in M_{\varepsilon}}\left|u_{\varepsilon}(x)\right| \leqq C<+\infty,
$$

where $S_{\varepsilon}$ is the set of positive solutions of (1.2) which minimize (1.1) $)_{\varepsilon}$.

Next we treat the asymptotic behaviours of $\lambda(\varepsilon)$ and positive solutions $u_{\varepsilon}$

Received January 25, 1994 ; revised August 19, 1994. 
of (1.2) which minimize $(1.1)_{\varepsilon}$. We put

$$
\lambda(0)=\inf _{X} \int_{M}|\nabla u|^{2} d x,
$$

where

$$
X=\left\{u \in H_{0}^{1}(M) ;\|u\|_{L^{p+1}(M)}=1, \quad u \geqq 0 \text { in } M\right\} .
$$

Then, there exists at least one solution $u_{0}$ which attains (1.3) and satisfies

$$
\begin{gathered}
-\Delta u_{0}=\lambda(0) u_{0}{ }^{p} \quad \text { in } M \\
u_{0}=0 \text { on } \partial M .
\end{gathered}
$$

We have the following theorems.

THEOREM 2. Fix $p \in(1,5)$. Then, there exists a constant $C$ independent of $\varepsilon$ such that

$$
|\lambda(\varepsilon)-\lambda(0)| \leqq C \varepsilon^{1 / 2}
$$

holds for any sufficiently small $\varepsilon>0$.

THEOREM 3. Fix $p \in(1,5)$. Assume that the minimizer $u_{0}$ of $(1.3)$ is unique. Then,

$$
\sup _{x \in M_{\varepsilon}}\left|u_{\varepsilon}(x)-u_{0}(x)\right| \longrightarrow 0 \quad \text { as } \quad \varepsilon \longrightarrow 0
$$

holds for any $u_{\varepsilon} \in S_{\varepsilon}$.

Remarks. When $M$ is a bounded domain in $\boldsymbol{R}^{2}$, Theorem 1 is proved in Ozawa-Roppongi [10].

When $M$ is a ball, the uniqueness of the minimizer of (1.3) is shown in Gidas, $\mathrm{Ni}$, and Nirenberg [4]. See also Dancer [2]. On the other hand, we do not know whether the minimizer $u_{\varepsilon}$ of $(1.1)_{\varepsilon}$ is unique or not in general and even in the case when $M$ is a ball. When the Robin boundary condition on $\partial B_{\varepsilon}$ in (1.2) is replaced by the zero Dirichlet condition, the uniqueness of $u_{\varepsilon}$ is proved in Dancer [3] for any sufficiently small $\varepsilon>0$ under the assumptions that the minimizer $u_{0}$ of (1.3) is unique, and that $\operatorname{Ker}\left(\Delta+p \lambda(0) u_{0}^{p-1}\right)=\{0\}$.

For related topics, the reader may be referred to Lin [5], Osawa-Ozawa [6], Ozawa [7], [8], [9].

Section 2 contains preliminary material. We give the proof of Theorems 1,2 and 3 in sections 3,4 and 5, respectively. In Appendix we give an extension lemma for a function on $M_{\varepsilon}$ to $M$. We will follow the established practice of using the same letter $C$ (with or without subscript) to denote different constants independent of $\varepsilon$. 


\section{Preliminary lemmas}

LEMMA 2.1. Fix $\xi \in(0,1)$ and $\alpha \in H^{\xi}\left(S^{2}\right)$. Then, there exists at least one solution of

$$
\begin{gathered}
\Delta v_{\varepsilon}(x)=0 \quad x \in \boldsymbol{R}^{3} \backslash \bar{B}_{\varepsilon} \\
\frac{\partial v_{\varepsilon}}{\partial \nu_{x}}(x)+k v_{\varepsilon}(x)=\alpha(\boldsymbol{\omega}), \quad x=w+\varepsilon \boldsymbol{\omega} \in \partial B_{\varepsilon}\left(\boldsymbol{\omega} \in S^{2}\right)
\end{gathered}
$$

satisfying

$$
\max _{x \in M_{\varepsilon}}\left|v_{\varepsilon}(x)\right| \leqq C \varepsilon\|\alpha\|_{H^{\xi}}\left(S^{2}\right) .
$$

Proof. Without loss of generality, we may assume that $w=0$. We put $x=r \omega\left(\omega \in S^{2}\right)$ and $\omega=(\sin \theta \cos \varphi, \sin \theta \sin \varphi, \cos \theta)(0 \leqq \theta<\pi, 0 \leqq \varphi<2 \pi)$. Let $P_{n}(z)$ be the Legendre polynomial and $P_{n}^{m}(z)$ be the associated Legendre function, that is,

$$
P_{n}^{m}(z)=\left(1-z^{2}\right)^{m / 2} \frac{d^{m}}{d z^{m}} P_{n}(z), \quad(|z| \leqq 1,0 \leqq m \leqq n) .
$$

It is well known that $\left\{P_{n}^{m}(\cos \theta) \cos m \varphi, P_{n}^{m}(\cos \theta) \sin m \varphi ; 0 \leqq m \leqq n\right\}_{n=0}^{\infty}$ is a complete orthogonal system of $L^{2}\left(S^{2}\right)$ consisting of eigenfunction of the LaplaceBeltrami operator $\Delta_{S}{ }^{2}$ whose eigenvalues are $-n(n+1), n=0,1,2, \cdots$.

Furthermore, we have the Parseval relation

$$
\begin{aligned}
& \sum_{n=0}^{\infty}(2 n+1)^{-1}\left(a_{n, 0}^{2}+\sum_{m=1}^{n}((n+m) ! / 2(n-m) !)\left(a_{n, m}^{2}+b_{n, m}^{2}\right)\right) \\
& =(4 \pi)^{-1}\|\alpha\|_{L 2(S 2)}^{2}
\end{aligned}
$$

for $\alpha(\omega)$ with the Fourier expansion

$$
\alpha(\omega)=\sum_{n=0}^{\infty} Y_{n}(\theta, \varphi),
$$

where

$$
Y_{n}(\theta, \varphi)=\sum_{n=0}^{\infty}\left(a_{n, m} \cos m \varphi+b_{n, m} \sin m \varphi\right) P_{n}^{m}(\cos \theta)
$$

We put

$$
v_{\varepsilon}(x)=\sum_{n=0}^{\infty}\left(\sum_{m=0}^{n}\left(s_{n, m} \cos m \varphi+t_{n, m} \sin m \varphi\right) P_{n}^{m}(\cos \theta)\right) r^{-(n+1)} .
$$

We see that

$$
\frac{\partial v_{\varepsilon}}{\partial \nu_{x}}(x)+k v_{\varepsilon}(x)_{1 x \in \partial B_{\varepsilon}}=\alpha(\boldsymbol{\omega})
$$




$$
=\sum_{n=0}^{\infty}\left(\sum_{m=0}^{n}\left(a_{n, m} \cos m \varphi+b_{n, m} \sin m \varphi\right) P_{n}^{m}(\cos \theta)\right)
$$

implies

$$
\left\{\begin{array}{l}
a_{n, m}=\varepsilon^{-(n+2)}(n+1+k \varepsilon) s_{n, m} \\
b_{n, m}=\varepsilon^{-(n+2)}(n+1+k \varepsilon) t_{n, m}
\end{array}\right.
$$

for $0 \leqq m \leqq n, n \geqq 0$. Then we have

$$
v_{\varepsilon}(x)=\varepsilon \sum_{n=0}^{\infty}(\varepsilon / r)^{n+1}(n+1+k \varepsilon)^{-1} Y_{n}(\theta, \varphi),
$$

and it satisfies (2.1) and (2.2). By (2.5), and by using the Schwarz inequality and the relation

$$
P_{n}(\cos \theta)^{2}+\sum_{m=1}^{n}(2(n-m) ! /(n+m) !) P_{n}^{m}(\cos \theta)^{2}=1
$$

we see that

$$
\left.\left|Y_{n}(\theta, \varphi)\right|^{2} \leqq a_{n, m}^{2}+\sum_{m=1}^{n}(n+m) ! / 2(n-m) !\right)\left(a_{n, m}^{2}+b_{n, m}^{2}\right) .
$$

From (2.6) and (2.7), we have

$$
\left|v_{\varepsilon}(x)\right| \leqq C \varepsilon^{2} r^{-1}\left(\sum_{n=0}^{\infty}(\varepsilon / r)^{2 n}(n+1)^{-1-\xi}\right)^{1 / 2} K(\xi)^{1 / 2} \leqq C_{\xi} \varepsilon K(\xi)^{1 / 2}
$$

for $x \in M_{\varepsilon}, \xi \in(0,1)$, where

$$
K(\xi)=\sum_{n=0}^{\infty}(2 n+1)^{-1} n^{\xi}\left(a_{n, 0}^{2}+\sum_{m=1}^{n}((n+m) ! / 2(n-m) !)\left(a_{n, m}^{2}+b_{n, m}^{2}\right)\right) .
$$

By (2.4) and observing that $j$-th eigenvalue of $-\Delta_{S^{2}} \sim C_{\jmath}$ as $\jmath \rightarrow \infty$, we can easily see that $K(\xi)^{1 / 2}$ is equivalent to the norm $\|\alpha\|_{H^{\xi}}{ }^{\left(S^{2}\right)}$. Thus we get (2.3) from $(2.8)$.

q.e.d.

By Lemma 2.1 and the same repeating construction of the function $v_{\varepsilon}^{(n)}$ as in Ozawa [7, Proposition 1, pp. 260-262], we have the following.

LEMMA 2.2. Fix $\xi \in(0,1)$. Assume that $u_{\varepsilon} \in C^{\infty}\left(M_{\varepsilon}\right)$ is harmonic in $M_{\varepsilon}, u_{\varepsilon}$ $=0$ on $\partial M$ and

$$
\frac{\partial u_{\varepsilon}}{\partial \nu_{x}}(x)+k u_{\varepsilon}(x)=L(\omega) \quad x=w+\varepsilon \omega \in \partial B_{\varepsilon}\left(\omega \in S^{2}\right)
$$

Then,

$$
\left\|u_{\varepsilon}\right\|_{L^{\infty}\left(M_{\varepsilon}\right)} \leqq C \varepsilon\|L\|_{H^{\xi}\left(S^{2}\right)}
$$

holds.

Next we want to show the following. 
Lemma 2.3. Fix $q \in[3 / 2,2]$ and let $\xi=2-(3 / q)$. Then,

$$
\|u(\varepsilon \cdot)\|_{H^{\xi}(S 2)} \leqq C_{q} \varepsilon^{1-(3 / q)}\|u\|_{W^{1}, q_{(M)}}
$$

holds for any $u \in W^{1, q}(M)$.

Here $\|u(\varepsilon \cdot)\|_{H} \xi_{\left(S^{2}\right)}$ denotes the $H^{\xi}\left(S^{2}\right)$-norm of the function $u(\varepsilon \omega)\left(\omega \in S^{2}\right)$.

Proof. Fix $q \in[3 / 2,2]$ and let $\xi$ be as above. Then, the Sobolev embedding: $W^{1, q}\left(\boldsymbol{R}^{3}\right) \subset W^{(1 / 2)+\xi, 2}\left(\boldsymbol{R}^{3}\right)$ holds (see, for example, Adams [1, Theorem 7.58, p. 218]). Since the trace operator: $W^{(1 / 2)+\xi, 2}\left(\boldsymbol{R}^{3}\right) \rightarrow H^{\xi}\left(S^{2}\right)$ is continuous,

$$
\|v\|_{H} \hat{\varepsilon}_{\left(S^{2}\right)} \leqq C\|v\|_{W^{1}, q_{(R 3)}}
$$

holds for any $v \in W^{1, q}\left(\boldsymbol{R}^{3}\right)$.

We take an arbitrary $u \in W^{1, q}(M)$ and take $\varphi \in C^{\infty}\left(\boldsymbol{R}^{3}\right)$ satisfying $0 \leqq \varphi \leqq 1$, $\varphi \equiv 1$ on $B_{2 \varepsilon}, \varphi \equiv 0$ on $\boldsymbol{R}^{3} \backslash \bar{B}_{3 \varepsilon}$ and $|\nabla \varphi| \leqq C \varepsilon^{-1}$. We put $v_{\varepsilon}(x)=u(\varepsilon x) \varphi(\varepsilon x)$. Then, $v_{\varepsilon} \in W_{0}^{1, q}\left(B_{3}\right)$. We extend $v_{\varepsilon}$ to $\boldsymbol{R}^{3}$ by defining $v_{\varepsilon}=0$ on $\boldsymbol{R}^{3} \backslash B_{3}$. Then, $v_{3} \in$ $W^{1, q}\left(\boldsymbol{R}^{3}\right)$ and

$$
\begin{aligned}
\left\|v_{\varepsilon}\right\|_{L}^{q} q_{\left(R^{3}\right)} & =\int_{B_{3}}|u(\varepsilon x) \varphi(\varepsilon x)|^{q} d x \\
& =\varepsilon^{-3} \int_{B_{3 \varepsilon}}|u(y) \varphi(y)|^{q} d y \leqq \varepsilon^{-3} \int_{B_{3 \varepsilon}}|u(y)|^{q} d y .
\end{aligned}
$$

Here we used the transformation of co-ordinates: $y=\varepsilon x$. Let $r=3 q /(3-q)$. Then, by the Sobolev embedding, $\|u\|_{L^{r}(M)} \leqq C\|u\|_{W^{1}, q_{(M)}}$ holds. Using Hölder's inequality, we have

$$
\begin{aligned}
\int_{B_{3 \boldsymbol{\varepsilon}}}|u(y)|^{q} d y & \leqq\left(\int_{B_{3 \varepsilon}}|u(y)|^{r} d y\right)^{q / r}\left(\int_{B_{3 \boldsymbol{\varepsilon}}} 1^{3 / q} d y\right)^{q / 3} \\
& \leqq C \varepsilon^{q}\|u\|_{L^{q}(M)}^{q} \leqq C \varepsilon^{q}\|u\|_{W^{1}, q_{(M)}}^{q}
\end{aligned}
$$

Therefore,

$$
\left\|v_{\varepsilon}\right\|_{L}^{q} q_{\left(R^{3}\right)} \leqq C \varepsilon^{q-3}\|u\|_{W^{1,}, q_{(, Y)}}^{q}
$$

holds. $C \varepsilon^{-1}$,

On the other hand, $\left|\nabla v_{\varepsilon}(x)\right|=\varepsilon \varphi(\varepsilon x)(\nabla u)(\varepsilon x)+\varepsilon u(\varepsilon x)(\nabla \varphi)(\varepsilon x)$ and $|\nabla \varphi| \leqq$

$$
\begin{aligned}
\left\|\nabla v_{\varepsilon}\right\|_{L}^{q} q_{\left(R^{3}\right)} & \leqq C \varepsilon^{q} \int_{B_{3}}|(\nabla u)(\varepsilon x)|^{q} d x+C \int_{B_{3}}|u(\varepsilon x)|^{q} d x \\
& \leqq C \varepsilon^{q-3} \int_{B_{3 \varepsilon}}|(\nabla u)(y)|^{q} d y+C \varepsilon^{-3} \int_{B_{3 \varepsilon}}|u(y)|^{q} d y
\end{aligned}
$$

hold. Using (2.11) in the second term of the right hand side of the above inequality, we have 


$$
\left\|\nabla v_{\varepsilon}\right\|_{L}^{q} q_{\left(R^{3}\right)} \leqq C \varepsilon^{q-3}\|\nabla u\|_{L^{q}(M)}^{q}+C \varepsilon^{q-3}\|u\|_{W^{1}, q_{(M)}}^{q_{1}} \leqq C \varepsilon^{q-3}\|v\|_{W^{1}, q_{(M)}^{q}}^{q} .
$$

From (2.12) and (2.13),

$$
\left\|v_{\varepsilon}\right\|_{W^{1}, q_{\left(R^{3}\right)}} \leqq C \varepsilon^{1-(3 / q)}
$$

holds.

Notice that $v_{\varepsilon}(x)=u(\varepsilon x)$ for $x \in S^{2}$. Therefore, by (2.14) and using (2.10) with $v=v_{\varepsilon}$, we can get (2.9).

q.e.d.

\section{Proof of Theorem 1}

Let $G_{\varepsilon}(x, y)$ be the Green function of the Laplacian in $M_{\varepsilon}$ satisfying

$$
\begin{aligned}
& -\Delta_{x} G_{\varepsilon}(x, y)=\delta(x-y), \quad x, y \in M_{\varepsilon} \\
& G_{\varepsilon}(x, y)=0, \quad x \in \partial M, y \in M_{\varepsilon} \\
& \frac{\partial}{\partial \nu_{x}} G_{\varepsilon}(x, y)+k G_{\varepsilon}(x, y)=0, \quad x \in \partial B_{\varepsilon}, y \in M_{\varepsilon},
\end{aligned}
$$

Let $G(x, y)$ be the Green function of the Laplacian in $M$ under the zero Dirichlet condition on $\partial M$. We put

$$
\begin{aligned}
& (G f)(x)=\int_{M} G(x, y) f(y) d y, \\
& \left(G_{\varepsilon} f\right)(x)=\int_{M_{\varepsilon}} G_{\varepsilon}(x, y) f(y) d y .
\end{aligned}
$$

For the sake of simplicity we write $\|\cdot\|_{L^{r}(M)},\|\cdot\|_{L^{r}\left(M_{\varepsilon}\right)}$ as $\|\cdot\|_{r},\|\cdot\|_{r, \varepsilon}$, respectively for $r \in[1, \infty]$.

We have the following.

LEMMA 3.1. Fix $q \in(3 / 2,2]$ and $f \in L^{q}\left(M_{\varepsilon}\right)$. Then,

$$
\left\|G_{\varepsilon} f-G \tilde{f}\right\|_{\infty, \varepsilon} \leqq C \varepsilon^{2-(3 / q)}\|f\|_{q, \varepsilon}
$$

holds. Here $\tilde{f}$ denotes the extension of $f$ to $M$ in Appendix of this paper.

Proof. Without loss of generality we may assume that $w=0$. We put $v_{\varepsilon}(x)=\left(G_{\varepsilon} f-G \tilde{f}\right)(x)$ for $x \in M_{\varepsilon}$. Then, $\Delta v_{\varepsilon}=0$ in $M_{\varepsilon}, v_{\varepsilon}=0$ on $\partial M$ and

$$
\left(\frac{\partial v_{\varepsilon}}{\partial \nu_{x}}+k v_{\varepsilon}\right)(x)=-\left(\frac{\partial}{\partial \nu_{x}} G \tilde{f}+k G \tilde{f}\right)(\varepsilon \omega) \quad x=\varepsilon \omega \in \partial B_{\varepsilon}\left(\omega \in S^{2}\right) .
$$

Let $\xi$ be as in Lemma 2.3. Then $\xi \in(0,1)$. Thus, by Lemmas 2.2 and 2.3,

$$
\left\|v_{\varepsilon}\right\|_{\infty, \varepsilon} \leqq C \varepsilon\left\|\left(\frac{\partial}{\partial \nu_{x}} G \tilde{f}+k G \tilde{f}\right)(\varepsilon \cdot)\right\|_{H \xi_{\left(S^{2}\right)}},
$$




$$
\|G \tilde{f}(\varepsilon \cdot)\|_{H} \boldsymbol{\xi}_{\left(S^{2}\right)} \leqq C \varepsilon^{1-(3 / q)}\|G \tilde{f}\|_{W^{1}, q_{(M)}}
$$

and

$$
\|\nabla G \tilde{f}(\varepsilon \cdot)\|_{H} \boldsymbol{\xi}_{\left(S^{2}\right)} \leqq\left(\varepsilon^{1-(3 / 4)}\|\nabla G \tilde{f}\|_{W^{1}, q(M)}\right.
$$

hold.

Since

$$
\begin{gathered}
\left(\frac{\partial}{\partial \nu_{x}} G \tilde{f}\right)(\varepsilon \omega)=\nu_{x} \cdot(\nabla G \tilde{f})(\varepsilon \omega)=-\omega \cdot(\nabla G \tilde{f})(\varepsilon \omega) \quad x=\varepsilon \omega \in \partial B_{\varepsilon}\left(\omega \in S^{2}\right), \\
\left|\left(\frac{\partial}{\partial \nu_{x}} G \tilde{f}\right)(\varepsilon \omega)\right| \leqq|(\nabla G \tilde{f})(\varepsilon \omega)|
\end{gathered}
$$

and

$$
\begin{aligned}
& \left|\left(\frac{\partial}{\partial \nu_{x}} G \tilde{f}\right)(\varepsilon \omega)-\left(\frac{\partial}{\partial \nu_{x}} G \tilde{f}\right)\left(\varepsilon \omega^{\prime}\right)\right| \\
= & \left|\omega \cdot(\nabla G \tilde{f})(\varepsilon \omega)-\omega^{\prime} \cdot(\nabla G \tilde{f})\left(\varepsilon \omega^{\prime}\right)\right| \\
= & \left|\omega \cdot\left\{(\nabla G \tilde{f})(\varepsilon \omega)-(\nabla G \tilde{f})\left(\varepsilon \omega^{\prime}\right)\right\}+\left(\omega-\omega^{\prime}\right) \cdot(\nabla G \tilde{f})\left(\varepsilon \omega^{\prime}\right)\right| \\
\leqq & \left|(\nabla G \tilde{f})(\varepsilon \omega)-(\nabla G \tilde{f})\left(\varepsilon \omega^{\prime}\right)\right|+\left|\omega-\omega^{\prime}\right|\left|(\nabla G \tilde{f})\left(\varepsilon \omega^{\prime}\right)\right|
\end{aligned}
$$

hold for any $\omega, \omega^{\prime} \in S^{2}$. Thus we have

$$
\begin{aligned}
& \left\|\left(\frac{\partial}{\partial \nu_{x}} G \tilde{f}\right)(\varepsilon \cdot)\right\|_{H^{\xi}\left(S^{2}\right)}^{2} \\
= & \int_{S^{2}}\left|\left(\frac{\partial}{\partial \nu_{x}} G \tilde{f}\right)(\varepsilon \omega)\right|^{2} d \omega+\iint_{S^{2} \times S^{2}}\left|\left(\frac{\partial}{\partial \nu_{x}} G \tilde{f}\right)(\varepsilon \omega)-\left(\frac{\partial}{\partial \nu_{x}} G \tilde{f}\right)\left(\varepsilon \omega^{\prime}\right)\right|^{2} \\
\cdot & \left|\omega-\omega^{\prime}\right|^{-2-2 \xi} d \omega d \omega^{\prime} \\
\leqq & \int_{S^{2}}|(\nabla G \tilde{f})(\varepsilon \omega)|^{2} d \omega+2 \iint_{S^{2} \times S^{2}}\left|(\nabla G \tilde{f})\left(\varepsilon \omega^{\prime}\right)\right|^{2}\left|\omega-\omega^{\prime}\right|^{-2 \xi} d \omega d \omega^{\prime} \\
& +2 \iint_{S^{2 \times S^{2}}}\left|(\nabla G \tilde{f})(\varepsilon \omega)-(\nabla G \tilde{f})\left(\varepsilon \omega^{\prime}\right)\right|^{2}\left|\omega-\omega^{\prime}\right|^{-2-2 \xi} d \omega d \omega^{\prime}
\end{aligned}
$$

Since $\xi \in(0,1)$, we can easily see

$$
\int_{S^{2}}\left|\omega-\omega^{\prime}\right|^{-2 \xi} d \omega=\frac{4^{\xi}}{1-\xi} \pi
$$

for any $\omega^{\prime} \in S^{2}$. From (3.5) and (3.6), we have

(3.7) $\left\|\left(\frac{\partial}{\partial \nu_{x}} G \tilde{f}\right)(\varepsilon \cdot)\right\|_{H_{\left(S^{2}\right)}^{2}}^{2}$

$$
\leqq C_{\xi}\left(\int_{S^{2}}|(\nabla G \tilde{f})(\varepsilon \omega)|^{2} d \omega+\iint_{S^{2} \times S^{2}}\left|(\nabla G \tilde{f})(\varepsilon \omega)-(\nabla G \tilde{f})\left(\varepsilon \omega^{\prime}\right)\right|^{2}\left|\omega-\omega^{\prime}\right|^{-2-2 \xi} d \omega d \omega^{\prime}\right)
$$


$=C_{\xi}\|(\nabla G \tilde{f})(\varepsilon \cdot)\|_{H}^{2} \xi_{\left(S^{2}\right)}$.

Notice that $\|G \tilde{f}\|_{W^{2}, q_{(M)}} \leqq C\|\tilde{f}\|_{q} \leqq C\|f\|_{q, \varepsilon}$ hold by a prior estimate and Lemma $\mathrm{A}$ in Appendix. Thus, by (3.3), (3.4) and (3.7),

$$
\begin{aligned}
& \left\|\left(\frac{\partial}{\partial \nu_{x}} G \tilde{f}+k G \tilde{f}\right)(\varepsilon \cdot)\right\|_{H^{\xi}\left(S^{2}\right)} \\
\leqq & C \varepsilon^{1-(3 / q)}\left(\|\nabla G \tilde{f}\|_{W^{1,}{ }_{(M)}}+k\|G \tilde{f}\|_{\left.W^{1,} q_{(M)}\right)}\right. \\
\leqq & C \varepsilon^{1-(3 / q)}\|G \tilde{f}\|_{W^{2}, q_{(M)}} \leqq C \varepsilon^{1-(3 / q)}\|f\|_{q, \varepsilon}
\end{aligned}
$$

hold. From (3.2) and (3.8), we get (3.1).

q.e.d.

Now we are in a position to prove Theorem 1 . We take an arbitrary $u_{\varepsilon} \in$ $S_{\varepsilon}$. We fix $q \in(3 / 2,2]$. Then, by the Sobolev embedding: $W^{2, q}(M) \subset C(\bar{M})$ and a priori estimate,

$$
\left\|G \tilde{u}_{\varepsilon}^{p}\right\|_{\infty, \varepsilon} \leqq C\left\|G \tilde{u}_{\varepsilon}^{p}\right\|_{W^{2}, q_{(M)} \leqq C\left\|\tilde{u}_{\varepsilon}^{p}\right\|_{q}}
$$

hold. Notice that $u_{\varepsilon}=\lambda(\varepsilon) G_{\varepsilon} u_{\varepsilon}^{p}$ and $0 \leqq \lambda(\varepsilon) \leqq C$. Thus, by Lemma 3.1 and (3.9), we have

$$
\begin{aligned}
\left\|u_{\varepsilon}\right\|_{\infty, \varepsilon} & \leqq\left\|\lambda(\varepsilon)\left(G_{\varepsilon} u_{\varepsilon}^{p}-G \tilde{u}_{\varepsilon}^{p}\right)+\lambda(\varepsilon) G \tilde{u}_{\varepsilon}^{p}\right\|_{\infty, \varepsilon} \\
& \leqq C\left\|G_{\varepsilon} u_{\varepsilon}^{p}-G \tilde{u}_{\varepsilon}^{p}\right\|_{\infty, \varepsilon}+C\left\|G \tilde{u}_{\varepsilon}^{p}\right\|_{\infty, \varepsilon} \\
& \leqq C\left(\varepsilon^{2-(3 / q)}+1\right)\left\|\tilde{u}_{\varepsilon}^{p}\right\|_{q, \varepsilon} \leqq C\left\|u_{\varepsilon}\right\|_{p q, \varepsilon}^{p} .
\end{aligned}
$$

At first we treat the case $p \in(1,2)$. We put $q=(p+1) / p$. Then, $q \in(3 / 2,2)$. We recall that $\left\|u_{\varepsilon}\right\|_{p+1, \varepsilon}=1$. Therefore, by (3.10), $\left\|u_{\varepsilon}\right\|_{\infty, \varepsilon} \leqq C\left\|u_{\varepsilon}\right\|_{p+1, \varepsilon}^{p}=C$ hold.

Next we treat the case $p \in[2,5)$. Since $(p+1) /(p-1)>3 / 2$, we can take $q \in(3 / 2,2]$ so that $(p+1) /(p-1)>q$. Notice that $q>3 / 2>(p+1) / p$. Thus we have the interpolation inequality:

$$
\left\|u_{\varepsilon}\right\|_{p q, \varepsilon} \leqq\left\|u_{\varepsilon}\right\|_{p+1, \varepsilon}^{a} \cdot\left\|u_{\varepsilon}\right\|_{\infty, \varepsilon}^{1-a},
$$

where $a=(p+1) /(p q)$. By (3.10), (3.11) and the fact that $\left\|u_{\varepsilon}\right\|_{p+1, \varepsilon}=1$,

$$
\left\|u_{\varepsilon}\right\|_{\infty, \varepsilon} \leqq C\left\|u_{\varepsilon}\right\|_{p q, \varepsilon}^{p} \leqq C\left\|u_{\varepsilon}\right\|_{\infty, \varepsilon}^{\tau}
$$

hold for $\tau=(1-a) p=p-(p+1) / q$. Since $(p+1)>(p-1)>q, \tau<1$ holds. This implies $\left\|u_{\varepsilon}\right\|_{\infty, \varepsilon} \leqq C$.

Thus we get the desired Theorem 1 .

Remark. Since $\left\|u_{\varepsilon}\right\|_{\infty, \varepsilon} \leqq C$ holds, we have the following by using Lemma 3.1 with $f=u_{\varepsilon}^{p}$ and $q=2$.

$$
\left\|G_{\varepsilon} u_{\varepsilon}^{p}-G \tilde{u}_{\varepsilon}^{p}\right\|_{\infty, \varepsilon} \leqq C \varepsilon^{1 / 2}
$$




\section{Proof of Theorem 2}

Since $u_{0} \cdot\left\|u_{0}\right\|{ }_{p+1, \varepsilon}^{-1} \in X_{\varepsilon}$, we see

$$
\lambda(\varepsilon) \leqq\left\|u_{0}\right\|_{p+1, \varepsilon}^{-2}\left(\int_{M_{\varepsilon}}\left|\nabla u_{0}\right|^{2} d x+k \int_{\partial B_{\varepsilon}} u_{0}^{2} d \sigma\right)
$$

by $(1.1)_{\varepsilon}$. Notice that $u_{0} \in C^{1}(\bar{M}),\left\|u_{0}\right\|_{p+1, \varepsilon}=1$ and $\lambda(0)=\left\|\nabla u_{0}\right\|_{2}^{2}$. Therefore,

$$
\begin{gathered}
\left\|u_{0}\right\|_{p+1, \varepsilon}^{p+1}=1-\int_{B_{\varepsilon}} u_{0}^{p+1} d x=1+O\left(\varepsilon^{3}\right), \\
\int_{M_{\varepsilon}}\left|\nabla u_{0}\right|^{2} d x=\lambda(0)-\int_{B_{\varepsilon}}\left|\nabla u_{0}\right|^{2} d x=\lambda(0)+O\left(\varepsilon^{3}\right),
\end{gathered}
$$

and

$$
\int_{\partial B_{\varepsilon}} u_{0}^{2} d \sigma=O\left(\varepsilon^{2}\right)
$$

hold. Summing up (4.1), (4.2), (4.3) and (4.4), we have the following.

$$
\lambda(\varepsilon) \leqq \lambda(0)+C\left(k \varepsilon^{2}+\varepsilon^{3}\right)
$$

We take $\phi_{\varepsilon} \in C^{\infty}\left(\boldsymbol{R}^{3}\right)$ satisfying $0 \leqq \phi_{\varepsilon} \leqq 1, \phi_{\varepsilon}=1$ on $\boldsymbol{R}^{3} \backslash B_{2 \varepsilon}, \phi_{\varepsilon}=0$ on $B_{\varepsilon}$, and $\left|\nabla \phi_{\varepsilon}\right| \leqq C \varepsilon^{-1}$. Since $\left(\phi_{\varepsilon} u_{\varepsilon}\right) \cdot\left\|\phi_{\varepsilon} u_{\varepsilon}\right\|_{p+1}^{-1} \in X$, we see

$$
\lambda(0) \leqq\left\|\psi_{\varepsilon} u_{\varepsilon}\right\|_{p+1}^{-2} \int_{M}\left|\nabla\left(\phi_{\varepsilon} u_{\varepsilon}\right)\right|^{2} d x
$$

by (1.3). We recall that $\left\|u_{\varepsilon}\right\|_{p+1, \varepsilon}=1$. Thus, we have

$$
\left\|\phi_{\varepsilon} u_{\varepsilon}\right\|_{p+1}^{p+1}=\int_{M_{\varepsilon}} u_{\varepsilon}^{p+1} d x+\int_{M_{\varepsilon}}\left(\phi_{\varepsilon}^{p+1}-1\right) u_{\varepsilon}^{p+1} d x=1+O\left(\varepsilon^{3}\right) .
$$

On the other hand, we see

$$
\int_{M}\left|\nabla\left(\psi_{\varepsilon} u_{\varepsilon}\right)\right|^{2} d x=I_{1}(\varepsilon)+I_{2}(\varepsilon)+I_{3}(\varepsilon),
$$

where

$$
\begin{aligned}
& I_{1}(\varepsilon)=\int_{M} \phi_{\varepsilon}^{2}\left|\nabla u_{\varepsilon}\right|^{2} d x, \\
& I_{2}(\varepsilon)=2 \int_{M} \phi_{\varepsilon} u_{\varepsilon} \nabla \psi_{\varepsilon} \cdot \nabla u_{\varepsilon} d x, \\
& I_{3}(\varepsilon)=\int_{M} u_{\varepsilon}^{2}\left|\nabla \psi_{\varepsilon}\right|^{2} d x .
\end{aligned}
$$

We recall $(1.1)_{\varepsilon}$ and Theorem 1 . Thus, we have 


$$
I_{1}(\varepsilon) \leqq \int_{M_{\varepsilon}}\left|\nabla u_{\varepsilon}\right|^{2} d x=\lambda(\varepsilon)-k \int_{\partial_{B_{\varepsilon}}} u_{\varepsilon}^{2} d \sigma \leqq \lambda(\varepsilon) \leqq C,
$$

$I_{3}(\varepsilon) \leqq C \varepsilon$ and $\left|I_{2}(\varepsilon)\right| \leqq\left\{I_{1}(\varepsilon) I_{3}(\varepsilon)\right\}^{1 / 2} \leqq C \varepsilon^{1 / 2}$. Summing up these facts, we have

$$
\int_{M}\left|\nabla\left(\psi_{\varepsilon} u_{\varepsilon}\right)\right|^{2} d x=\lambda(\varepsilon)+O\left(\varepsilon^{1 / 2}\right) .
$$

From (4.6), (4.7) and (4.8), we see that $\lambda(0) \leqq \lambda(\varepsilon)+C \varepsilon^{1 / 2}$. Combining this with (4.5), we get Theorem 2 .

\section{Proof of Theorem 3}

At first we want to show the following.

LEMMA 5.1. Let $\tilde{u}_{\varepsilon}$ be an extension of $u_{\varepsilon}$ to $M$ as in Appendix. Assume that the minimizer $u_{0}$ of (1.3) is unique. Then,

$$
\tilde{u}_{\varepsilon} \longrightarrow u_{0} \text { strongly in } H_{0}^{1}(M) \text { as } \varepsilon \longrightarrow 0 \text {. }
$$

Proof. Since $\tilde{u}_{\varepsilon}=u_{0}$ a.e. in $M_{\varepsilon}$,

$$
\left\|\tilde{u}_{\varepsilon}\right\|_{p+1}^{p+1}=\left\|u_{\varepsilon}\right\|_{p+1}^{p+1}+\int_{B_{\varepsilon}} u_{\varepsilon}^{p+1} d x=1+O\left(\varepsilon^{3}\right)
$$

and

$$
\int_{M_{\varepsilon}}\left|\nabla \tilde{u}_{\varepsilon}\right|^{2} d x=\int_{M_{\varepsilon}}\left|\nabla u_{\varepsilon}\right|^{2} d x+\int_{B_{\varepsilon}}\left|\nabla \tilde{u}_{\varepsilon}\right|^{2} d x
$$

hold. By $(1.1)_{\varepsilon}$, Theorems 1 and 2, we see

$$
\int_{M_{\varepsilon}}\left|\nabla u_{\varepsilon}\right|^{2} d x=\lambda(\varepsilon)-k \int_{\partial B_{\varepsilon}} u_{\varepsilon}^{2} d \sigma=\lambda(0)+O\left(\varepsilon^{1 / 2}\right) .
$$

On the other hand, $\left\|\nabla \tilde{u}_{\varepsilon}\right\|_{L^{2}(M)}^{{ }^{2}(} \leqq C$ holds from Theorem 1 and (A.3) of Lemma $A$ in Appendix. Thus, we have

$$
\int_{B_{\varepsilon}}\left|\nabla \tilde{u}_{\varepsilon}\right|^{2} d x=0(1) \quad \text { as } \quad \varepsilon \longrightarrow 0 .
$$

Summing up these facts, we get the following.

$$
\begin{aligned}
& \left\|\tilde{u}_{\varepsilon}\right\|_{p+1} \longrightarrow 1 \quad \text { as } \varepsilon \longrightarrow 0 \\
& \left\|\nabla \tilde{u}_{\varepsilon}\right\|_{2}^{2} \longrightarrow\left\|\nabla u_{0}\right\|_{2}^{2}=\lambda(0) \quad \text { as } \varepsilon \longrightarrow 0
\end{aligned}
$$

Next we want to show the following.

$$
\tilde{u}_{\varepsilon} \longrightarrow u_{0} \text { weakly in } H_{0}^{1}(M) \text { as } \varepsilon \longrightarrow 0
$$

Assume that (5.3) does not hold. Then, there exist $\eta>0, F \in\left(H_{0}^{1}(M)\right)^{*}$, and a 
sequence $\left\{\varepsilon_{n}\right\}_{n=0}^{\infty}$ satisfying $\varepsilon_{n} \downarrow 0(n \rightarrow \infty)$ such that

$$
\left|F\left(\tilde{u}_{\varepsilon_{n}}\right)-F\left(u_{0}\right)\right| \geqq \eta
$$

holds. Since $\left\{\tilde{u}_{\varepsilon_{n}}\right\}$ is bounded in $H_{0}^{1}(M)$, there exist a subsequence $\left\{\tilde{u}_{\varepsilon_{n^{\prime}}}\right\}$ and $v \in H_{0}^{1}(M)$ satisfing

$$
\begin{array}{ll}
\tilde{u}_{\varepsilon_{n}} \longrightarrow v & \text { weakly in } H_{0}^{1}(M) \\
\tilde{u}_{\varepsilon_{n}} \longrightarrow v & \text { strongly in } L^{p+1}(M) \\
\tilde{u}_{\varepsilon_{n}} \longrightarrow v & \text { a.e. in } M .
\end{array}
$$

Since $\tilde{u}_{\varepsilon_{n^{\prime}}} \geqq 0$ a.e. in $M, v \geqq 0$ a.e. in $M$. From (5.1) and (5.2), $\left\|\tilde{u}_{\varepsilon_{n}}\right\|_{p_{+1} \rightarrow 1}$ and $\left\|\nabla \tilde{u}_{\varepsilon_{n}}\right\|_{2}^{2} \rightarrow\left\|\nabla u_{0}\right\|_{2}^{2}=\lambda(0)$ as $n^{\prime} \rightarrow \infty$. Thus, by (5.5), we have $\|v\|_{p+1}=1$ and

$$
\|\nabla v\|_{2} \leqq \liminf _{n^{\prime} \rightarrow \infty}\left\|\nabla \tilde{u}_{\varepsilon_{n^{\prime}}}\right\|_{2} \leqq\left\|\nabla u_{0}\right\|_{2}=\lambda(0)^{1 / 2} .
$$

Here we used the lower semi-continuity of the $H_{0}^{1}$-norm. Therefore we have $v \in X$ and $\lambda(0) \leqq\|\nabla v\|_{2}^{2} \leqq\left\|\nabla u_{0}\right\|_{2}^{2} \leqq \lambda(0)$. Hence $v$ is a minimizer of (1.3). Thus, $v=u_{0}$ must hold by the assumption. Letting $n=n^{\prime} \rightarrow \infty$ in (5.4), we have $0=$ $\left|F(v)-F\left(u_{0}\right)\right| \geqq \eta$. This contradicts $\eta>0$. Therefore we get (5.3).

From (5.2), (5.3) and the uniform convexity of $H_{0}^{1}$, we get the desired result. q.e.d.

Now we are in a position to prove Theorem 3. Since $u_{s}=\lambda(\varepsilon) G_{\varepsilon} u_{s}{ }^{p}$ and $u_{0}=\lambda(0) G u_{0}{ }^{p}$ hold, we have

$$
u_{\varepsilon}(x)-u_{0}(x)=\sum_{i=1}^{3} J_{i}(\varepsilon ; x) \quad x \in M_{\varepsilon},
$$

where

$$
\begin{aligned}
& J_{1}(\varepsilon ; x)=\lambda(\varepsilon)\left(G_{\varepsilon} u_{\varepsilon}^{p}-G \tilde{u}_{\varepsilon}^{p}\right)(x), \\
& J_{2}(\varepsilon ; x)=\lambda(\varepsilon) G\left(\tilde{u}_{\varepsilon}^{p}-u_{0}^{p}\right)(x), \\
& J_{3}(\varepsilon ; x)=(\lambda(\varepsilon)-\lambda(0)) G u_{0}^{p}(x) .
\end{aligned}
$$

We recall that $0<\lambda(\varepsilon) \leqq C$. Thus, by $(3.12)$ and Theorem $2,\left\|J_{1}(\varepsilon ; \cdot)\right\|_{\infty, \varepsilon} \leqq C \varepsilon^{1 / 2}$ and $\left\|J_{3}(\varepsilon: \cdot)\right\|_{\infty, \varepsilon} \leqq C \varepsilon^{1 / 2}\left\|G u_{0}^{p}\right\|_{\infty, \varepsilon} \leqq C \varepsilon^{1 / 2}$ hold. Furthermore, by the Sobolev embedding: $W^{2,6}(M) \subset C^{0}(M)$ and a priori estimate,

$$
\left\|G\left(\tilde{u}_{\varepsilon}^{p}-u_{0}^{p}\right)\right\|_{\infty} \leqq C\left\|G\left(\tilde{u}_{\varepsilon}^{p}-u_{0}^{p}\right)\right\|_{W^{2},{ }^{6}(M)} \leqq C\left\|\tilde{u}_{\varepsilon}^{p}-u_{0}^{p}\right\|_{L^{6}(M)}
$$

hold. Thus, by using Theorem 1 and Lemma 5.1,

$$
\begin{aligned}
\left\|J_{2}(\varepsilon ; \cdot)\right\|_{\infty, \varepsilon} & \leqq C\left\|\tilde{u}_{\varepsilon}^{p}-u_{0}^{p}\right\|_{L^{6}(M)} \\
& \leqq C\left\|\tilde{u}_{\varepsilon}-u_{0}\right\|_{L^{6}(M)} \sup _{\varepsilon>0} \max \left(\left\|u_{0}\right\|_{\infty}^{p-1},\left\|\tilde{u}_{\varepsilon}\right\|_{\infty}^{p-1}\right)
\end{aligned}
$$




$$
\leqq C\left\|\tilde{u}_{\varepsilon}-u_{0}\right\|_{H_{0}^{1}(M)}=o(1) .
$$

Summing up these facts, we get the desired Theorem 3.

\section{Appendix}

Let $M, M_{\varepsilon}$ be as in Introduction. Then we have the following.

LEMMA A. For a function $u$ on $M_{\varepsilon}$, there exists a function $\tilde{u}$ satisfying the following:

$$
\begin{aligned}
& \tilde{u}(x)=u(x) \text { a.e. in } M_{\varepsilon}, \\
& \|\tilde{u}\|_{L^{s}(M)} \leqq C\|u\|_{L^{s}\left(M_{\varepsilon}\right)} \quad(1 \leqq s \leqq \infty)
\end{aligned}
$$

holds for any $u \in L^{s}\left(M_{\varepsilon}\right)$.

$$
\|\tilde{u}\|_{I^{1}(M)} \leqq C\|u\|_{H^{1}\left(M_{\varepsilon}\right)}+C \varepsilon^{1 / 2}\|u\|_{L^{\infty}\left(M_{\varepsilon}\right)}
$$

holds for any $u \in H^{1}\left(M_{\varepsilon}\right) \cap L^{\infty}\left(M_{\varepsilon}\right)$.

Proof. Without loss of generality, we may assume that $w=0$. For a function $u$ on $M_{\varepsilon}$, we put

$$
\tilde{u}(x)=\left\{\begin{array}{l}
u(x) \quad x \in M_{\varepsilon} \\
u\left(\varepsilon^{2} x|x|^{-2}\right) \eta_{\varepsilon}(x) \quad x \in B_{\varepsilon},
\end{array}\right.
$$

where $\eta_{\varepsilon}(x) \in C^{\infty}\left(\boldsymbol{R}^{3}\right)$ satisfies $0 \leqq \eta_{\varepsilon} \leqq 1, \eta_{\varepsilon}=1$ on $\boldsymbol{R}^{3} \backslash \bar{B}_{\varepsilon / 2}, \eta_{\varepsilon}=0$ on $B_{\varepsilon / 4}$ and $\left|\nabla \eta_{\varepsilon}\right| \leqq 8 \varepsilon^{-1}$. Notice that both $\eta_{\varepsilon}\left(\varepsilon^{2} x|x|^{-2}\right)$ and $\left(\nabla \eta_{\varepsilon}\right)\left(\varepsilon^{2} x|x|^{-2}\right)$ vanish on $\boldsymbol{R}^{3} \backslash B_{4 \varepsilon}$. Then, by using the Kelvin transformation of co-ordinates : $y=\varepsilon^{2} x|x|^{-2}$, we have

$$
\begin{aligned}
\int_{B_{\varepsilon}}|\tilde{u}(x)|^{s} d x & =\int_{R^{3} \backslash B_{\varepsilon}}|u(y)|^{s} \eta_{\varepsilon}\left(\varepsilon^{2} y|y|^{-2}\right)^{s}\left(\varepsilon|y|^{-1}\right)^{6} d y \\
& \leqq \int_{M_{\varepsilon}}|u(y)|^{s} d y \quad(1 \leqq s<\infty),
\end{aligned}
$$

where the term $\left(\varepsilon|y|^{-1}\right)^{6}$ comes from the absolute value of the determinant of the Jacobian of the Kelvin transformation. And we have

$$
\begin{aligned}
\int_{B_{\varepsilon}}|\nabla \tilde{u}(x)|^{2} d x= & C \int_{B_{\varepsilon}}\left|u\left(\varepsilon^{2} x|x|^{-2}\right)\right|^{2}\left|\left(\nabla \eta_{\varepsilon}\right)(x)\right|^{2} d x \\
& +C \int_{B_{\varepsilon}}\left(\varepsilon|x|^{-1}\right)^{4}\left|(\nabla u)\left(\varepsilon^{2} x|x|^{-2}\right)\right|^{2}\left|\eta_{\varepsilon}(x)\right|^{2} d x \\
& \leqq C \varepsilon^{4} \int_{M_{\varepsilon}}|u(y)|^{2}|y|^{-6} d y+C \int_{M_{\varepsilon}}|(\nabla u)(y)|^{2} d y
\end{aligned}
$$




$$
\leqq C \varepsilon\|u\|_{L^{\infty}\left(M_{\varepsilon}\right)}^{2}+C \int_{M_{\varepsilon}}|(\nabla u)(y)|^{2} d y .
$$

Thus we get the desired result.

q.e.d.

\title{
REFERENCES
}

[1] R. Adams, Sobolev Spaces, Academic Press, New York, 1975.

[2] E.N. DANCER, The effect of domain shape on the number of positive solutions of certain nonlinear equations, J. Differential Equations, 74 (1988), 120-156.

[3] E.N. DANCER, On the positive solutions of some weakly nonlinear equations on annular region, Math. Z., 206 (1991), 551-562.

[4] B. Gidas, W.M. Ni ANd L. NirenberG, Symmetry and related properties via the maximum principle, Comm. Math. Phys., 68 (1979), 209-243.

[5] S.S. Lin, Semilinear elliptic equation on singularly perturbed domains, Comm. Partial Differential Equations, 16 (1991), 617-645.

[6] T. Osawa And S. Ozawa, Singular variation of nonlinear eigenvalues, Proc. Japan Acad., 69A (1993), 217-218.

[7] S. OzAwA, Spectra of domains with small spherical Neumann boundary, J. Fac. Sci. Univ. Tokyo Sect. IA, 30 (1983), 259-277.

[8] S. OzAwA, $L^{\infty}$ boundedness of nonlinear eigenfunction under singular variation of domains, preprint.

[9] S. OzAwA, Singular variation of the ground state eigenvalue for a sem-ilinear elliptic equation, Tôhoku Math. J., 45 (1993), 359-368.

[10] S. Ozawa AND S. Roppongi, Singular variation of domains and $L^{\infty}$ boundedness of eigenfunction for some semi-linear elliptic equations, Proc. Japan Acad., 70A (1994), 67-70.

\author{
Department of Mathematics \\ Faculty of Science \\ Tokyo Institute of Technology \\ Oh-okayama, Meguro-ku, Tokyo, 152 \\ JAPAN
}

